\title{
Optimal Location of Novel Robotic Prostrate Cancer Biopsy and Brachytherapy Treatment Devices
}

\author{
Sina Firouzy ${ }^{1}$, Dylan Jones ${ }^{2}$ and Ashraf Labib ${ }^{1}$ \\ ${ }^{1}$ Faculty of Business and Law, University of Portsmouth, Portsmouth, UK \\ ${ }^{2}$ School of Mathematics and Physics, University of Portsmouth, Portsmouth, UK \\ \{sina.firouzy, dylan.jones, ashraf.labib\}@port.ac.uk
}

\begin{abstract}
This paper details research towards the optimal placement of a novel robotic device for the detection and treatment of prostate cancer, via biopsy and brachytherapy respectively. A methodology for analysis of available data in order to determine geographical areas with relatively high prevalence of prostate cancer and low access to treatment is proposed. Areas in the South of the UK with high values of these indices are highlighted. The development of single and multiple criteria optimization models based on the new metric in order to optimally locate a small number of future prototype treatment devices is discussed. Discussions, conclusions and avenues for future research are given.
\end{abstract}

Keywords: Healthcare Technologies, Cancer detection and treatment, Optimization.

\section{Introduction}

This study focuses on finding the optimal locations for a small number of prototype robotic devices in the South of the United Kingdom for the detection and treatment of prostate cancer. This problem is considered as part of a project for the development of the robotic device, funded by the European Union 2-Seas Interreg scheme under the CoBra (Co-operative Brachytherapy) [1] project.

The remainder of this paper is divided into three sections which describe the data analysis (Section 2) and and model formulation and solution (Sections 3), and conclusions and suggestions for future work (Section 4.

\section{Data analysis}

\subsection{Identification of the Prostate cancer Occurrence Hotspot}

Two distinct quantities can be used to assess the prevalence of prostate cancer, namely prostate cancer incidence, and prostate cancer mortality rate. Prostate cancer incidence 
represent the number of patients (per year) who are diagnosed with prostate cancer. The diagnosis methods used are not consistent throughout the UK, and the efficacy of different methods are different; this renders incidence rate unsuitable for comparing prostate cancer prevalence in different areas of the UK. Therefore, mortality rate is a better measure for this purpose [2], and is used in this analysis. The only risk factors of prostate cancer currently known are age and ethnicity. Deprivation does not seem to affect the risk of prostate cancer [2,3]. A quantitative breakdown of the risk is available, for age groups with 5 year increments [3], which has been used in this study. The risk is very low before the age of 50 , after which the mortality rate monotonically increases with age.

To exclude the effect of different age profiles in different populations, prostate cancer mortality rates are commonly expressed as Age Standard (AS) rates, which reveal the real differences between populations which can be attributed to factors other than age.

Ethnicity is the other factor relevant to the risk of prostate cancer. Compared to white males, Age-Standard (AS) prostate cancer mortality rate is significantly lower in Asian men, and significantly higher in Black males. For this reason, the ethnic demographics of the population may have to be taken into account in order to obtain a reliable estimate of prostate cancer prevalence in different areas. However, a study of prostate cancer mortality rates in different areas of Britain (analyzed at ward ${ }^{1}$ level) revealed a flat distribution map; i.e. the AS mortality rate is quite uniform in the UK. This means that the differences in ethnical demographics of different areas within the UK can be ignored in estimating prostate cancer prevalence, and age demographics alone are sufficient for that purpose. Therefore, age has been used as the sole estimating factor in this study, as follows:

1. The geographic area of study (the southern part of England, in the 2 seas zone) was first broken down to the level of postcode district (i.e. PO2).

2. The age demographics of the population in each postcode district were then obtained from the Office of National Statistics [4]. The data contains the number of male residents of an area, within each age range. Each age range spans over five years (e.g. 50-54 years old).

3. The Age-Standard risk rate of prostate cancer for different age ranges [3] was used together with age demographics of each postcode district to produce an estimate. To do this, the number of the male population within each age range was multiplied by the prostate cancer rate corresponding to that age range, as follows:

$\sum_{i=1}^{n} M_{i} . P_{i}$

where $M_{i}$ and $P_{i}$ are the male population and the Prostate cancer rate for the i'th age group, respectively, and $\mathrm{n}$ is the number of age groups.

This was done for all age ranges, and the sum of all products represents the estimated rate of prostate cancer for the given postcode district.

4. The procedure was executed for all postcode district, to produce a map of prostate cancer dispersion in the area studied.

\footnotetext{
${ }^{1} \mathrm{~A}$ ward is a district level geographic division in The UK.
} 
The map of the area studied is shown in Fig. 1, together with the centroids of the postcode districts in the area of study. The centroids are significant because the distance from each postcode district (to a given treatment center) was measured from its centroid. The prostate cancer prevalence map developed in this study is represented in a color intensity graph in Fig. 2.
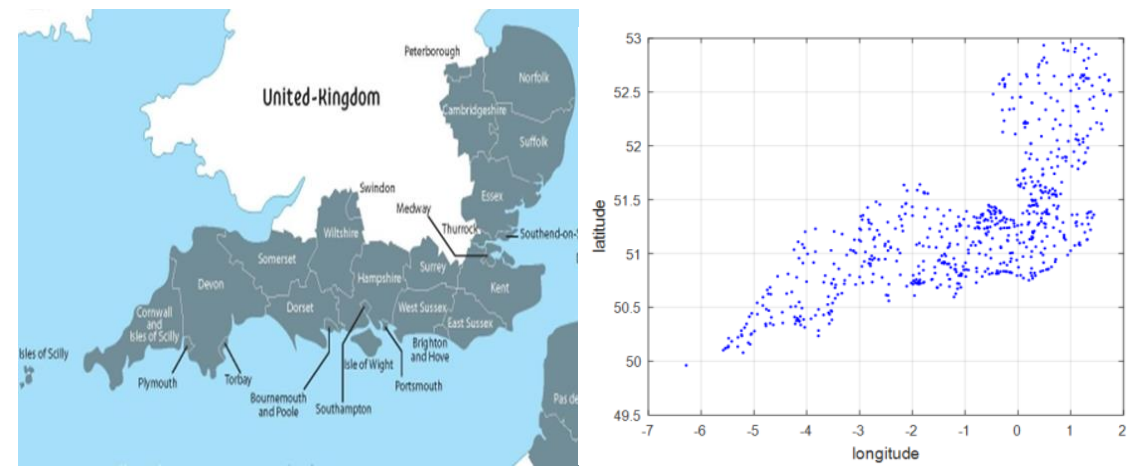

Fig. 1. The Southern parts of England within the 2 Seas area (left), and the centroids of the postcode districts within the 2 seas area in the UK (right)

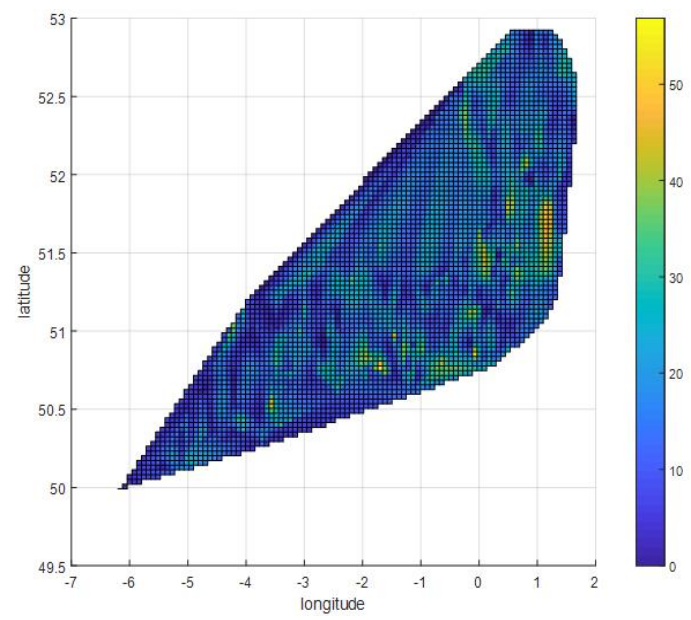

Fig. 2. The color-intensity graph of the estimated prostate cancer rate (patient/year) in each postcode district within the 2 seas zone in the UK

\subsection{Identification of the Prostate Cancer Treatment Dry-Spots}

The term dry here refers to a relative lack of access to treatment facilities, which has been measured by the distance from the considered area to the nearest prostate cancer treatment facilities. The current version of the analysis is based on the linear (Euclidean) distance rather than road distance, which is one of the limitations of this work. Fig. 3 shows the location of the treatment centers for prostate cancer. 


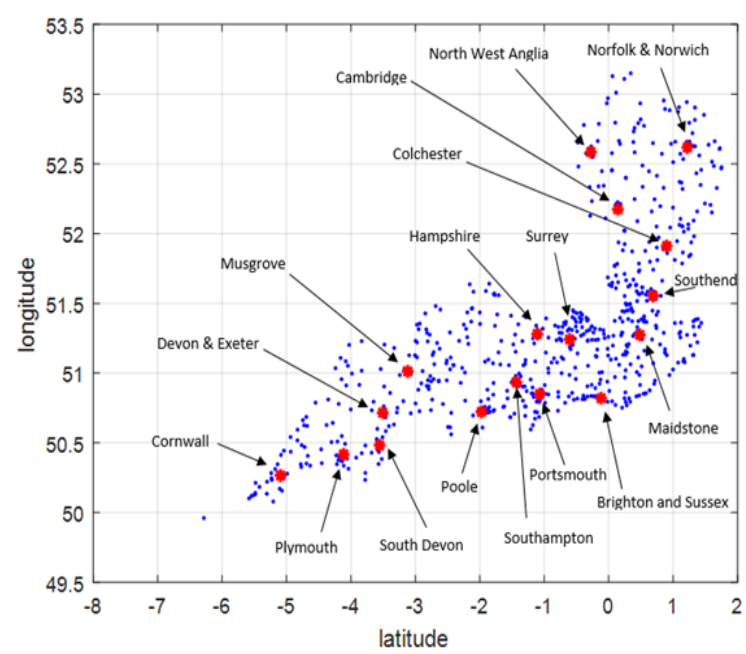

Fig. 3. The location of the treatment centers (red dots), and the centroids of the postcode districts within the 2seas area in the UK (blue dots)

\section{$3 \quad$ A strategy to find the optimal location}

The method used to solve the problem is as follwos:

1. For each of the potential hosting locations (the treatment centers shown in Fig. 3), a total travel time distance was defined, as follows:

a. For each of the postcode districts, the number of the patients in that district was multiplied by the distance to the given treatment center. The resultant value was calculated and saved for all of the postcode districts.

b. The sum of the above-mentioned value for all of the postcode districts was saved as the total travel distance for the given treatment center.

2. The total travel distance, explained above, was calculated for all of the treatment center.

3. The treatment center with the smallest value of the total travel distance was identified as the optimal location.

The optimization goal is to minimize the travel distance for all of the patients who may be potential receivers of the treatment provided by the new device. The result shows that the optimal location is the treatment center in Surrey, shown in .Fig. 3.

\section{Discussion and Conclusion}

In this paper, we have developed a methodology towards the development of an optimized facility location modelling that includes geographical areas with relatively high prevalence of prostate cancer against access to treatment centers. We have focused our 
application on the South of the UK. A detailed mapping has identified age as the dominant factor for the analysis. The development and combination of measures for incidence and ease of access allows the identification of priority postcode areas. These are useful from a policy perspective as they show the areas most in need of enhanced provision, and from a modelling perspective as they allow effective measurement criteria for the development of single and multiple criteria optimization models.

The suggested single and multiple criteria facility location model offers a promising platform for future modelling developments. These relate on a specific level to the European Union project in which the next generation prostate cancer detection and treatment devices are being developed. On a general level, the mapping and proposed optimization models in this paper can be seen as the basis as a wider methodology to identify priority geographical areas for placement of medical resources to detect and treat disease, particularly in the case of a relatively small amount of resources and a large potential treatment population.

Further modelling developments involve the development of multiple criteria facility location models for the allocation of the small number of robotic devices. These could relate to the improvement of access for specific target postcodes with (i) poor access (dryness), (ii) high incidence levels (hotness) or (iii) combination of the both as detailed by the methodology develop in this paper. Specific targets could be developed for these priority postcodes and for the general population with respect to access time, travel distance and associated patient costs. Trade-off with respect to priority versus general access and the cost of locating multiple devices could be explored via a multi-objective weight sensitivity analysis. Given the target-based and geographical nature of this problem, extended network goal programming [10] is a viable modelling methodology.

\section{References}

1. The CobRa Project Website. Available from: https://cobra2seas.eu/.

2. Hansell, A.L. and R.E. Ghosh, The Environment and Health Atlas for England and Wales. 2014: Oxford University Press.

3. Cancer Research UK. [cited 2018 November]; Available from: https://www.cancerresearchuk.org/health-professional/cancerstatistics/statistics-by-cancer-type/prostate-cancer.

4. Population Age Demographics of UK Postcodes, Table LC1117EW 12/2018]; Available from: https://www.nomisweb.co.uk/census/2011/lc1117ew. 